Monatsschr Kinderheilkd 2021 · 169:902-911 https://doi.org/10.1007/s00112-021-01239-0 Angenommen: 9. Juni 2021

Online publiziert: 13 . Juli 2021

๑) Springer Medizin Verlag GmbH, ein Teil von Springer Nature 2021

Redaktion

G. Hansen, Hannover

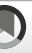

\section{Individualisierung der Diabetestherapie durch Automatisierung der Insulingabe}

\author{
T. Biester ${ }^{1} \cdot$ K. Dovc ${ }^{2,6} \cdot$ A. Chobot ${ }^{3} \cdot$ M. Tauschmann ${ }^{4} \cdot$ T. Kapellen $^{5}$ \\ ' AUF DER BULT, Kinder- und Jugendkrankenhaus, Hannover, Deutschland \\ ${ }^{2}$ Pädiatrische Diabetologie, Endokrinologie und Stoffwechselerkrankungen, UMC - \\ Universitätskinderklinik, Ljubljana, Slowenien \\ ${ }^{3}$ Medzinische Fakultät, Kinderklinik, Universität Opole, Opole, Polen \\ ${ }^{4}$ Universitätsklinik für Kinder- und Jugendheilkunde, Medizinische Universität Wien, Wien, Österreich \\ ${ }^{5}$ Kinderendokrinologe und Diabetologe, MEDIAN Kinderklinik „Am Nicolausholz“ Bad Kösen, Naumburg, \\ Deutschland \\ ${ }^{6}$ Medizinische Fakultät, Universität Ljubljana, Ljubljana, Slowenien
}

\section{Zusammenfassung}

\section{In diesem Beitrag}

- Automated insulin delivery

- Vollständig geschlossener Regelkreis

- Benachteiligung im Kleinkindalter

- Implikationen für die Nutzung von OpenSource-Technologien

- Therapieziele werden auf Technologie ausgerichtet

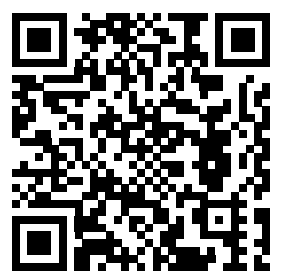

QR-Code scannen \& Beitrag online lesen

Insulinpumpen und Glucosesensoren haben sich in Registerdaten als effektiv in der Verbesserung der Diabetestherapie und Reduktion akuter Komplikationen gezeigt. In der pädiatrischen Diabetologie ist die Nutzung mindestens eines technischen Geräts Standard. Durch die Kombination beider Systeme ergibt sich Möglichkeit der automatischen Insulinabgabe („automated insulin delivery“, AID).

Viele AID-Systeme sind in klinischen Studien getestet und haben sich als sicher und effektiv erwiesen. Die Versorgungsituation in Deutschland erlaubt es derzeit nur, Mitgliedern der gesetzlichen Krankenversicherungen ein bestimmtes System zu verordnen; dieses ist für Kinder, die jünger als 7 Jahre sind, nicht geeignet. Gründe liegen in gesetzlichen Hürden und mangelnder Zertifizierung durch die Hersteller. Die CE-Zertifikate können Probleme bei der Insulinverordnung mit sich bringen. „Opensource"-Systeme sind Varianten, mit denen bestehende Regularien umgangen werden können. Daraus ergeben sich sowohl für Nutzer wie auch für Verordner Risiken.

Die dauerhafte Nutzung setzt sowohl auf Anwender- als auch auf Behandlerseite die fundierte Kenntnis der Eigenschaften der einzelnen AID-Systeme voraus. Eine vollständige Automatisierung funktioniert noch nicht. Zur Evaluation der AID-Therapie sind die metrischen Daten der Glucosesensoren, die „Zeit im Zielbereich" und der "Glucose Management Indicator" anerkannte und geeignete Parameter, da sie eine Beratung auf Basis der reellen Daten aus dem Alltag der Menschen mit Diabetes zulassen.

Da alle Glucosesensoren über Cloud-basierte Software ausgelesen werden oder die Daten automatisch aus einem telefonverbundenen Empfangsgerät beziehen, ist die ideale technische Grundlage für eine telemedizinische Betreuung geschaffen, die noch der Ausgestaltung bedarf.

\section{Schlüsselwörter}

Insulinpumpe · Glucosesensor · Insulininfusionssysteme · Zeit im Zielbereich · Telemedizin

\section{Einleitung}

Die häufigste zu behandelnde Stoffwechselerkrankung im Kindes- und Jugendalter ist der Diabetes mellitus Typ 1. Aufgrund der kontinuierlich steigenden jährlichen Rate an Neuerkrankungen sind im- mer mehr betroffene Kinder und Jugendliche zu verzeichnen [1].

Immer niedrigere $\mathrm{HbA}_{1 \mathrm{c}}$-Werte als Ausdruck der mittleren Stoffwechsellage sind erreichbar, ohne dass die Gefahr der Unterzuckerungen steigt [2]. Die Leitlinie der International Society for Pediatric and Ad- 
Hier steht eine Anzeige.

黑 Springer 


\begin{tabular}{|c|c|c|c|c|c|}
\hline & $\begin{array}{l}\text { t:slim X2 mit Control } \\
\text { IQ (Tandem, San } \\
\text { Diego, CA, USA) }\end{array}$ & $\begin{array}{l}\text { MiniMed 780G } \\
\text { (Medtronic } \\
\text { Inc, North- } \\
\text { ridge, CA, USA) }\end{array}$ & $\begin{array}{l}\text { MiniMed 770G } \\
\text { (Medtronic } \\
\text { Inc, North- } \\
\text { ridge, CA, USA) }\end{array}$ & $\begin{array}{l}\text { Diabeloop } \\
\text { DBLG1/DBL4T (Dia- } \\
\text { beloop SA, Grenoble, } \\
\text { Frankreich) }\end{array}$ & $\begin{array}{l}\text { CamAPS FX (CamDiab } \\
\text { Ltd., Cambrigde, UK) }\end{array}$ \\
\hline Insulinpumpe & T-Slim X:2 & MiniMed 780G & MiniMed 770G & Accu-Chek Insight & Dana R/S \\
\hline Glucosensor & DexCom G6 & Guardian 3 & Guardian 3 & DexCom G6 & DexCom G6 \\
\hline Sensordauer & 10 Tage & 7 Tage & 7 Tage & 10 Tage & 10 Tage \\
\hline $\begin{array}{l}\text { Anzahl der notwen- } \\
\text { digen Blutzucker- } \\
\text { messungen }\end{array}$ & Kein Minimum, selten & Mindestens 2-mal/Tag & $\begin{array}{l}\text { Mindestens 4- bis } \\
\text { 6-mal/Tag }\end{array}$ & Kein Minimum, selten & Kein Minimum, selten \\
\hline $\begin{array}{l}\text { Art des Algorith- } \\
\text { mus }\end{array}$ & MPC & $\begin{array}{l}\text { PID mit Fuzzy Logic } \\
\text { und MPC-Anteil }\end{array}$ & PID & MPC & MPC \\
\hline $\begin{array}{l}\text { Plattform des Algo- } \\
\text { rithmus }\end{array}$ & In der Pumpe & In der Pumpe & In der Pumpe & Handgerät & Android-Smartphone \\
\hline $\begin{array}{l}\text { Alterbeschränkung } \\
\text { laut Hersteller }\end{array}$ & $>6$ Jahre & > 7 Jahre & > 7 Jahre & $\begin{array}{l}\text { 12-18 Jahre (DBL4T) } \\
\text { > 18 Jahre (DBLG1) }\end{array}$ & $\begin{array}{l}>1 \mathrm{Jahr} \\
\text { (auch für Schwangere) }\end{array}$ \\
\hline $\begin{array}{l}\text { Glucosezielwert des } \\
\text { Systems }\end{array}$ & $110 \mathrm{mg} / \mathrm{dl}$ & $100 / 120 \mathrm{mg} / \mathrm{dl}$ & $120 \mathrm{mg} / \mathrm{dl}$ & $100-180 \mathrm{mg} / \mathrm{dl}$ & $80-200 \mathrm{mg} / \mathrm{dl}$ \\
\hline Insuline & $\begin{array}{l}\text { Humalog und NovoRa- } \\
\text { pid }\end{array}$ & $\begin{array}{l}\text { Humalog und NovoRa- } \\
\text { pid }\end{array}$ & $\begin{array}{l}\text { Humalog und NovoRa- } \\
\text { pid }\end{array}$ & NovoRapid & $\begin{array}{l}\text { Humalog und NovoRa- } \\
\text { pid, Fiasp }\end{array}$ \\
\hline $\begin{array}{l}\text { Automatische } \\
\text { Korrekturbolus- } \\
\text { gaben }\end{array}$ & $\mathrm{Ja}$ & $\mathrm{Ja}$ & Nein & $\mathrm{Ja}$ & Nein \\
\hline $\begin{array}{l}\text { Möglichkeit der } \\
\text { Datenverfügbarkeit }\end{array}$ & $\begin{array}{l}\text { Download, Diasend } \\
\text { (Sensordaten Clarity) }\end{array}$ & Automatisch, CareLink & Automatisch, CareLink & $\begin{array}{l}\text { Download, Diasend } \\
\text { (Sensordaten Clarity) }\end{array}$ & Automatisch, Diasend \\
\hline Sonstiges & Nachtmodus & Per Handy anzusehen & Per Handy anzusehen & $\begin{array}{l}\text { Variable „Aggressivi- } \\
\text { tät" }\end{array}$ & "Boost-Modus" \\
\hline $\begin{array}{l}\text { Temporäres Ziel } \\
\text { erhöhen }\end{array}$ & Aktivitätsmodus & $\begin{array}{l}\text { Temporäres Ziel } \\
(150 \mathrm{mg} / \mathrm{dl})\end{array}$ & $\begin{array}{l}\text { Temporäres Ziel } \\
(150 \mathrm{mg} / \mathrm{dl})\end{array}$ & $\begin{array}{l}\text { Zen-Modus } \\
\text { ( } 20-40 \mathrm{mg} / \mathrm{dl} \text { höher } \\
\text { als aktuelles Ziel) }\end{array}$ & $\begin{array}{l}\text { Aktivitätsziel: „ease-off } \\
\text { modus“ }\end{array}$ \\
\hline $\begin{array}{l}\text { Verfügbar in } \\
\text { Deutschland (Mai } \\
\text { 2021) }\end{array}$ & Nein & Nein & $\mathrm{Ja}$ & Als DBLG1 (> 18 Jahre) & $\begin{array}{l}\text { Ggf. bei privat Kran- } \\
\text { kenversicherten }\end{array}$ \\
\hline
\end{tabular}

olescent Diabetes (ISPAD) hat daher einen $\mathrm{HbA}_{1 \mathrm{c}}$-Therapiezielwert $<7 \%$ festgelegt, wenn den Patienten Möglichkeiten wie eine Insulinpumpe und ein Glucosesensor zur Verfügung stehen. Ein niedrigerer $\mathrm{HbA}_{1 \mathrm{c}}$-Wert ist auf lange Sicht gleichbedeutend mit einem geringeren Risiko für diabetesbedingte Folgeerkrankungen. Unverändert in den letzten Jahren bleibt das Risiko für eine diabetische Ketoacidose bei Manifestation; im Zuge des ersten Lockdowns der durch die "coronavirus disease 2019“ (COVID-19) ausgelösten Pandemie hat sich dieses Risiko bei Kleinkindern sogar verdoppelt [3]. Hier sind präventive Ansätze gefordert, um eine solche Entwicklung zu verhindern. Positive Beispiele gibt es z. B. aus dem Raum Stuttgart; die dortige Aufklärung bei der Einschulung hat zur Reduktion der Ketoacidoserate geführt [4].
Die Arbeitsgemeinschaft für Pädiatrische Diabetologie hat diese Maßnahme nun auf ganz Deutschland ausgeweitet (diabeteskinder.de).

\section{I) Pumpe und Sensor sind in der heutigen Zeit Standards in der Diabetestherapie bei Kindern und Jugendlichen}

Der wesentliche Fortschritt in der Behandlung von Menschen mit einem Typ-1-Diabetes besteht neben der Verabreichung neuer Insuline [5] in der stetigen Weiterentwicklung der technischen Möglichkeiten, bei der sich die Pädiatrie mit einem hohen Nutzungsgrad zeigt. Insulinpumpen und Glucosesensoren werden in allen Altersgruppen, die von Kinderärzten betreut werden, deutlich häufiger ange- wendet als von Erwachsenen; bei Kleinkindern beträgt die Nutzungsrate für die Insulinpumpe $>95 \%$ [6]. Die exponentielle Zunahme der Nutzung von Glucosesensoren mit aktuell $>70 \%$ aller pädiatrischen Patienten ist eine direkte Folge der Kostenübernahme durch die gesetzliche Krankenversicherung (bei Erfüllung der Indikationskriterien) im Jahr 2016. Mehr als 60\% aller Minderjährigen mit einem Typ-1-Diabetes nutzen eine Insulinpumpe: Pumpe und Sensor sind Therapiestandards.

Die Vorteile der Insulinpumpentherapie (20-fach feinere Dosierbarkeit als bei Pens, kontinuierliche Abgabe einer bis zu halbstündlich einstellbaren Basalrate, Rechenunterstützung durch integrierten Computer) mit denen eines Glucosesensors (kein Stechen zur erforderlichen Einzelmessung, sondern „immer" aktuelle 


\begin{tabular}{|c|c|c|c|c|c|}
\hline & CamAPS FX & Diabeloop & MiniMed 780G & MiniMed 770G & $\begin{array}{l}\text { T-Slim X:2 mit Con- } \\
\text { trol IQ }\end{array}$ \\
\hline $\begin{array}{l}\text { Veränderbare Einstel- } \\
\text { lungen }\end{array}$ & $\begin{array}{l}\text { Kohlenhydratfaktor } \\
\text { "Aggressivität“ } \\
\text { Glucosezielwert } \\
\text { Seit Neuestem Art der } \\
\text { Mahlzeit (fettig) }\end{array}$ & $\begin{array}{l}\text { Insulin-Tagesgesamt- } \\
\text { menge } \\
\text { Gewicht } \\
\text { Mittlere Tagesgesamt- } \\
\text { kohlenhydratmenge } \\
\text { "Aggressivität" } \\
\text { Glucosezielwert } \\
\text { Menge der empfohle- } \\
\text { nen Notfallkohlenhy- } \\
\text { drate }\end{array}$ & $\begin{array}{l}\text { Kohlenhydratfaktor } \\
\text { Insulinwirkdauer } \\
\text { Temporäres Ziel } \\
\text { Glucosezielwert }\end{array}$ & $\begin{array}{l}\text { Kohlenhydratfaktor } \\
\text { Insulinwirkdauer } \\
\text { Temporäres Ziel }\end{array}$ & $\begin{array}{l}\text { Basalrate } \\
\text { Kohlenhydratfaktor } \\
\text { Insulinwirkdauer } \\
\text { Temporäres Ziel } \\
\text { Tagesgesamtmenge } \\
\text { Schlafmodus } \\
\text { Sportmodus }\end{array}$ \\
\hline $\begin{array}{l}\text { Systemseitige Ein- } \\
\text { stellungen, die nicht } \\
\text { verändert werden } \\
\text { können }\end{array}$ & $\begin{array}{l}\text { Insulinwirkdauer } \\
\text { Basalrate }\end{array}$ & $\begin{array}{l}\text { Basalrate } \\
\text { Korrekturfaktor } \\
\text { Kohlenhydratfaktor }\end{array}$ & $\begin{array}{l}\text { Basalrate } \\
\text { Korrekturfaktor } \\
\text { Korrekturbolusgaben }\end{array}$ & $\begin{array}{l}\text { Basalrate } \\
\text { Korrekturfaktor } \\
\text { Korrekturbolusgaben }\end{array}$ & Insulinwirkdauer \\
\hline
\end{tabular}

Messwerte, Alarmfunktionen, Trendanzeige) zu kombinieren, ist also naheliegend. Außerdem entwickeln sich mit der Verwendung einer Insulinpumpe weniger akute Komplikationen [7], und es besteht ein Vorteil in ihrer frühen Anwendung [8].

Ein Gerät mit der Funktion der vorausschauenden Abschaltung der Insulinzufuhr durch eine Pumpen-Sensor-Kombination ist in Deutschland seit einigen Jahren verfügbar, seit dem vergangenen Jahr auch im zweiten, wissenschaftlich getesteten System.

Ein geschlossener Regelkreislauf, in der Forschung lange als "closed loop" bezeichnet, ist inzwischen als sog. automatische Insulinabgabe erhältlich (,automated insulin delivery", AID, [9]).

\section{Automated insulin delivery}

Da der Begriff "hybrid closed loop", der die automatische Insulinsteuerung mit Ausnahme der Mahlzeitenbolusgaben beschreibt, inzwischen mit einem bestimmten Produkt assoziiert ist, hat sich international AID als deskriptive Bezeichnung der teilautomatisierten Systeme zur Insulinapplikation durchgesetzt. Es handelt sich um automatisierte Steuerungssysteme, wie sie auch in der Industrie Anwendung finden:

1. Ein Messwert wird erhoben (Gewebsglucose).

2. Ein Programm errechnet die Differenz aus aktuellem Wert und Zielwert sowie die notwendige Änderung der abzugebenden Stellgröße (Insulinabgabe).
3. Das Insulin wird abgegeben und wirkt im Körper.

4. Der Kreislauf beginnt von vorn.

Der Rechenalgorithmus unterscheidet sich in den verschiedenen Modellen und damit der Art, wie die Insulindosis ermittelt wird. Die einzelnen mathematischen Details der Algorithmen sollen in diesem Beitrag nicht ausgeführt werden [10].

Je nach regulatorischem Stand der einzelnen Länder sind automatisierte Systeme erhältlich [11-14]. Eine Übersicht über die CE-zertifizierten Systeme in der Europäischen Union gibt 0 Tab. 1.

\section{I) Die AID-Systeme haben sich in klinischen Studien durch die erhöhte Zeit im Zielbereich ausgezeichnet}

In den klinischen Studien aller Systeme haben sich immer die gleichen Muster in den Ergebnissen gezeigt [12, 13, 15]:

- keine schwerwiegenden Komplikationen durch Überdosierung des Insulins,

- Senkung der mittleren Glucosewerte,

- höhere Senkung der mittleren Glucosewerte, je jünger die Kinder sind,

- Reduktion der Schwankungen,

- höherer Effekt in der Nacht,

- bessere Effektivität, wenn die Bolusgaben vor der Mahlzeit erfolgen,

- bei deutlicher manueller Insulinüberdosierung (Mahlzeiten) sind weiterhin Kohlenhydratgaben notwendig.

Im Vergleich zur ausschließlichen Nutzung von "Continuous-glucose-monitoring" (CGM)-Systemen oder vorausschauender
Abschaltung zeichnen sich AID-Systeme in klinischen Studien stets durch eine erhöhte Zeit im Zielbereich aus [16]. Die Ergebnisse wesentlicher klinischer Studien zeigt $\square$ Abb. 1.

Unterschiede bestehen in den für die Anwender "spürbaren“ Komponenten: Je nach System kann es sein, dass trotz eines Glucosesensors neben den Kalibrierungsmessungen auch präprandiale Blutzuckermessungen nötig sind. Andere Sensoren kommen laut Herstellerangabe fast ganz ohne „blutige Werte" aus. Manche Systeme sind per Bluetooth-Verbindung auf einem Handy aus der Ferne einzusehen und somit zu "überwachen“. Es gibt auch Systeme, bei denen der Steuerungsalgorithmus nicht in der Pumpe verbaut ist, sondern sich auf einem externen Gerät befindet ( $\bullet$ Tab. 1). Die Unterschiede der einzelnen Systeme, die Einstellungen von Anwendern oder Diabetesteams angepasst werden können (oder müssen), zeigt $\mathbf{0}$ Tab. 2.

Aufgrund der Vielfalt der Systeme ist ihre genaue Kenntnis notwendig, um die einzelnen Funktionen sowie die Vor- und Nachteile z.B. im Rahmen einer Diabetesberatung vor der Entscheidung für ein System gut zu erläutern, damit die Patienten eine fundierte Entscheidung auf Basis der persönlichen Schwerpunktsetzung treffen können.

Beispiele der in Deutschland genutzten Systeme zeigen die $\mathbf{A b b}$. 2, 3 und 4. Bei allen Systemen ist augenfällig, dass das Basalinsulin wellenförmig, durch den Algorithmus berechnet, abgegeben wird, infolgedessen verläuft die Glucosekurve insbesondere in der Nacht "glatt". 


\begin{tabular}{|l|l|l|l|l|l|l|l|}
\hline & $\begin{array}{l}\text { Control } \\
\text { IQ } a\end{array}$ & $\begin{array}{l}670 \\
\text { G } b\end{array}$ & DBLG1 $c$ & CamAPS $d$ & $3-7$ J. $e$ & $8-14$ J. $e$ & 28-35 J. $f$ \\
\hline $\begin{array}{l}\mathrm{HbA}_{1 c} \\
\text { baseline }\end{array}$ & 7.4 & 7.4 & 7.6 & 8.3 & 7.1 & 7.0 & 6.8 \\
\hline $\begin{array}{l}\mathrm{HbA} \\
\text { Delta \% }\end{array}$ & -0.33 & -0.50 & -0.15 & -0.36 & -0.50 & -0.7 & -0.5 \\
\hline $\begin{array}{l}\text { TIR 70-180 } \\
\text { OL }\end{array}$ & 59.1 & 66.7 & 59.4 & 54.0 & 70.8 & 77.2 & 68.0 \\
\hline $\begin{array}{l}\text { TIR 70-180 } \\
\text { CL }\end{array}$ & 71.0 & 72.2 & 68.5 & 65.0 & 78.6 & 82.4 & 79.3 \\
\hline TBR $<70$ OL & 2.2 & 5.9 & 4.3 & 3.9 & 4.0 & 3.4 & 2.5 \\
\hline TBR & 1.6 & 3.3 & 2.2 & 2.6 & 3.7 & 4.6 & 1.8 \\
\hline
\end{tabular}

Abb. $1<$ Ergebnisse der Zulassungsstudien aktueller Automated-insulin-delivery-Systeme (blau) sowie publizierte Studienergebnisse von "Open-source"-Systemen (grün) (kein Head-to-head Vergleich möglich, da unterschiedliche Kollektive und Studienprotokolle). $T I R$ "time in range", $T B R$ "time below range", $O L$ "open loop", $C L$ "closed loop". $a$ aus [27], $b$ aus [11], $c$ aus [26], $d$ aus [12], e aus [27], faus [28]

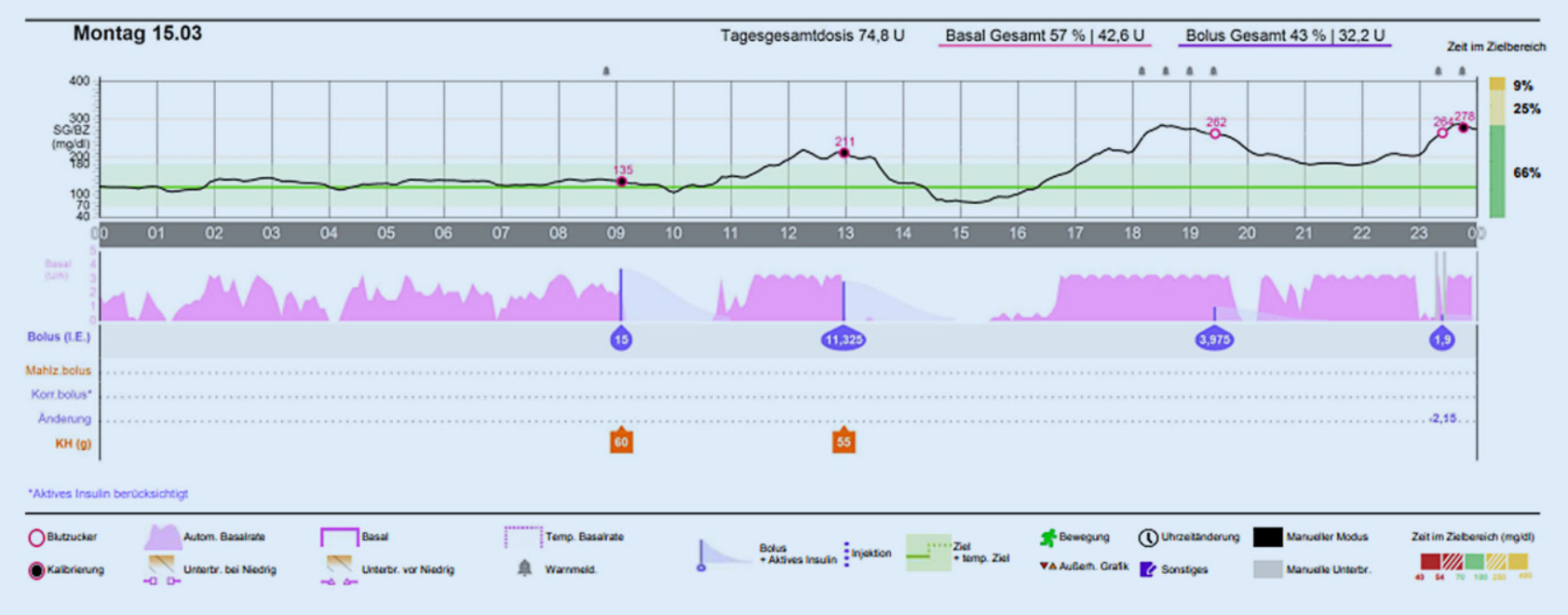

Abb. $2 \Delta$ Ein Tag im Hybrid-AID-Modus mit der Pumpe Minimed 670G. Die rosafarbene Fläche im unteren Abschnitt stellt die automatische Steuerung der Basalrate dar; bei ihrem Fehlen ist die Insulinzufuhr unterbrochen. Blaue Balken bilden manuelle Bolusgaben ab. Dargestellt ist eine stabile Glucosekurve in der Nacht bei variabler Insulinabgabe. Mahlzeitenbolusgabe vor dem Essen um 9 Uhr: kein postprandialer Anstieg. Mahlzeit ohne Bolusgabe um 17 Uhr: deutliche Hyperglykämie trotz automatischer Erhöhung der Basalinsulinabgabe. Grüne Linie: Glukosezielwert von $120 \mathrm{mg} / \mathrm{dl}$

Nach einer getroffenen Verordnung und Schulung auf das System der Wahl müssen die Nutzer nicht nur die Pumpen- und die Sensortherapie beherrschen, sondern auch die Anwendung und Besonderheiten des automatisieren Systems kennen, denn keines der aktuellen Systeme ist ein "Selbstläufer" (s. unten). Dieses kann u.U. schwierig sein. In den USA hat sich gezeigt, dass viele Erwachsene, aber auch Jugendliche die Automatisierung nach einem Jahr nicht mehr verwenden. Als ursächlich hat sich eine wahrscheinlich unzureichende Schulung gezeigt, aber auch Erwartungen an den Grad der Automatisierung, den aktuelle Systeme nicht erfüllen konnten [17]. International und auch in Deutschland [18] gibt es daher inzwischen Konzepte, wie die Umstellung und Schulung auf ein solches System erfolgen könnten, um die Adhärenz und somit auch den Effekt des Systems zu erhalten.

Die erste Studie, die 2 Hybrid-AID-Systeme verglichen hat, zeigt Vorteile für den Stoffwechsel bei dem System, dass permissiver bei hohen Werten ist, also die An- 


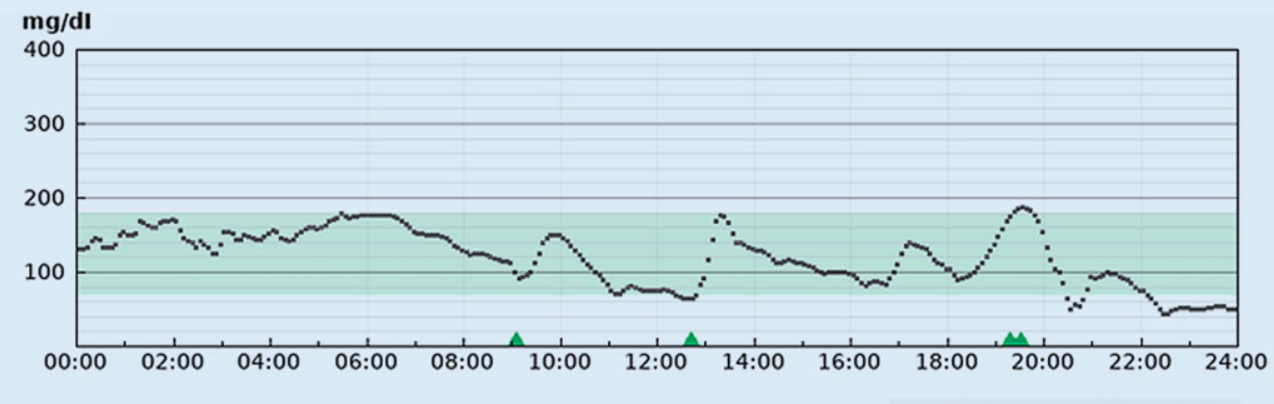

a

다욤

Basal (Einheiten pro Std)

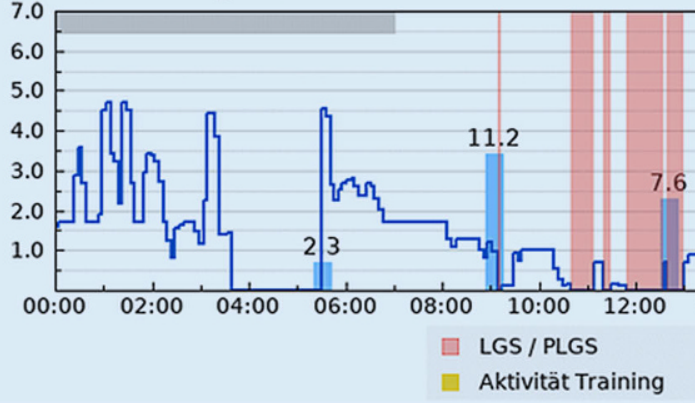

b a CGM $\Delta$ Kohlenhydrate

Bolus (Einheiten)

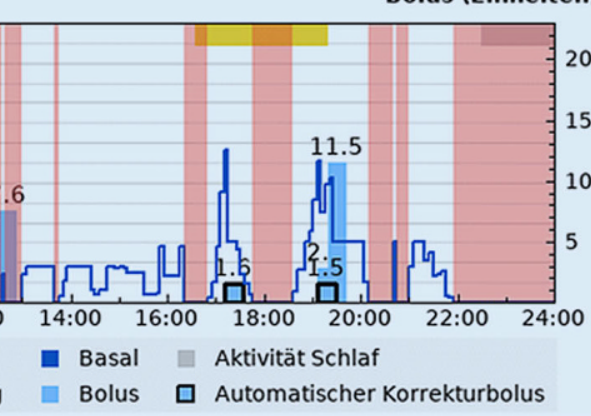

Abb. $3<$ Ein Tag im Hybrid-AID-Modus mit der Pumpe T:slim X2, ControlIQ-Steuerung (in Deutschland derzeit nur in klinischen Studien anwendbar). a Verlaufskurve des Glucosesensors. b Blaue Kurve: automatische Steuerung der Basalrate, rote Balken Unterbrechung der Insulinzufuhr bei prädiktierten Werten unter $80 \mathrm{mg} / \mathrm{dl}$. Blaue Balken ohne Rand manuelle Bolusgaben, $\mathrm{Bal}$ ken mit Rand automatische Korrekturbolusgaben

\section{$\mathbf{m g} / \mathbf{d l}$}

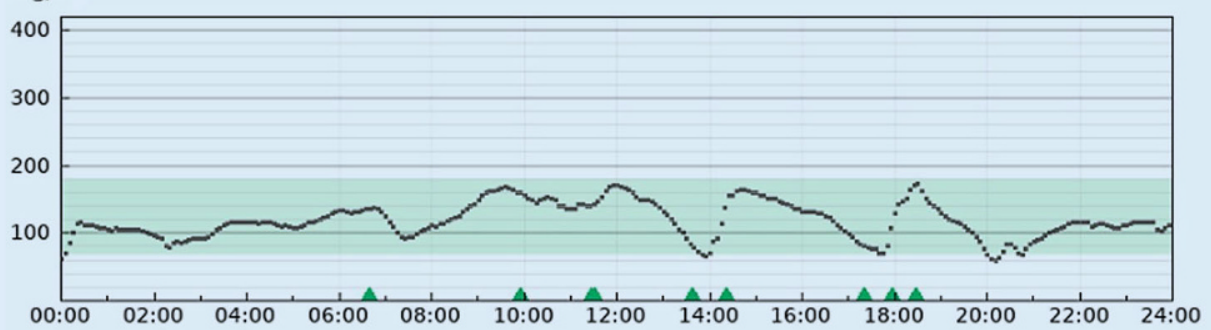

\section{Basal (Einheiten pro Std)}

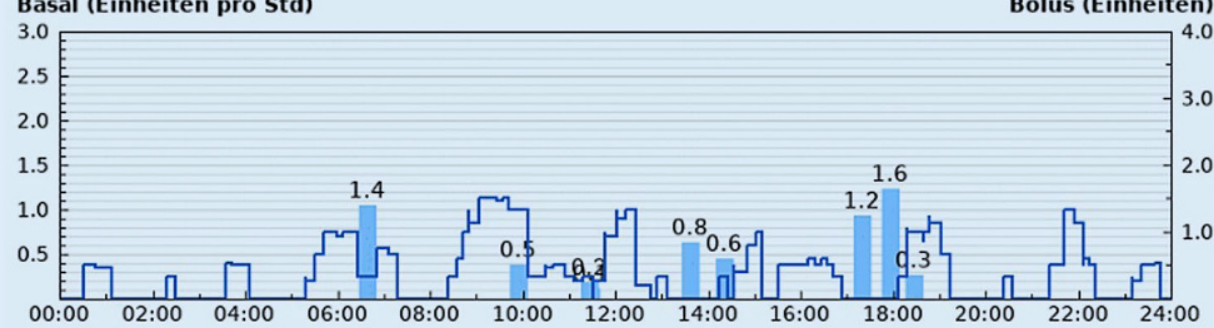

CGM

(O) Aktive CGM-Zeit: $100 \%$

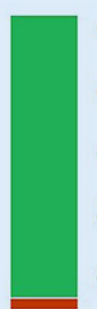

- $0 \%$ Sehr hoch (i)

$0 \%$ Hoch (i)

- $96 \%$ Zeit im Zielbereich (i)

- $4 \%$ Niedrig (i)

- $0 \%$ Sehr niedrig (i)

Zielbereich: $70-180 \mathrm{mg} / \mathrm{dl}$

Kohlenhydrate

Zeit

$06: 38$

09:55

11:27

11:30

13:38

$14: 22$

17:19

17:56

$18: 27$

b

- Basal Bolus

Abb. 4 ム Ein Tag im Hybrid-AID-Modus mit dem CamAPS Fx bei einem 6-jährigen Kind. a Glukosesensorkurve, b blaue Kurve im unteren Abschnitt automatische Steuerung der Basalrate, Balken manuelle Bolusgaben. (Aus Biester et al. [29]) 
wender weniger aus der Automatisierung „hinauswirft", und das über einen stärkeren Automatismus bei der Bolusabgabe verfügt, also Bolusabgaben auf der Basis von Sensorwerten anstatt Blutzuckermessungen durchführt [19].

\section{Vollständig geschlossener Regelkreis}

Ein „voller closed loop", also eine komplette, automatisierte Übernahme der Insulintherapie durch ein technisches System ist derzeit aus vielerlei Gründen nicht möglich. Die Anpassung der Insulintherapie basiert auf der Veränderung der Glucosesensorwerte. Bis postprandiale Konzentrationsanstiege der Gewebsglucose einen Gradienten ergeben, der auf eine Mahlzeitengabe rückschließen lässt, können 10-20 min vergehen. Anschließend wird das Insulin, das auch bei neuen, ultraschnell wirkenden Präparaten mindestens $10 \mathrm{~min}$ zur Resorption benötigt, s.c. verabreicht. Somit ergeben bei vollautomatischen Systemen nach dem Essen derzeit immer deutliche Peaks der Serumund Gewebsglucosekonzentration, die es eigentlich zu vermeiden gilt [20].

\section{Benachteiligung im Kleinkindalter}

Das derzeit einzige verfügbare System mit einer Hybrid-AID-Funktion, das von den gesetzlichen Krankenversicherungen (GKV) übernommen wird, ist erst für den Gebrauch im Patientenalter ab 7 Jahren geeignet. Im Gegensatz zum europäischen CE-Label ist dieses in den USA bereits für Patienten ab 2 Jahren zugelassen. Auch kommende Systeme sind erst ab einem höheren Alter anwendbar. Das verfügbare und $a b$ dem Alter von einem Jahr CEzertifizierte System CamAPS Fx ist derzeit nicht verordnungsfähig, somit können Kleinkinder in Deutschland nicht mit derart effizienten AID-Systemen behandelt werden wie größere Kinder. Und auch bei der Vorstufe, der prädiktiven Insulinabschaltung, gibt es derzeit nur ein Modell, das im Vorschulalter anwendbar ist.

Neben der Industrie, die dafür verantwortlich ist, ihre Produkte auch im Kleinkindalter zu prüfen und mit einem CELabel zu versehen, sind Organisationen von Betroffenen und Behandlern aufge- rufen, dieses einzufordern. Ein Grund für die derzeit noch nicht bestehende Verordnungsfähigkeit des CamAPS Fx ist die Begrenzung der gesetzlichen Verordnungsfähigkeit von Apps (digitale Gesundheitsanwendungen, DiGA) auf die Medizinproduktklassen I und Ila. Da eine AID-App Therapieänderungen automatisch vornehmen kann, gehört sie in die Gruppe der invasiveren Medizinprodukte Ilb. Diese wurden bei der Gesetzgebung nicht berücksichtigt.

\section{Implikationen für die Nutzung von Open-Source-Technologien}

Schon lange gibt es die Bestrebung auch vonseiten der Anwender, automatische Systeme zu nutzen zu können. Da die technischen Voraussetzungen solcher Systeme mit der Verbreitung leistungsfähiger Smartphones allgegenwärtig sind, liegt es nahe, dass kundige Nutzer selbst Systeme entwickeln. Aus dieser „\#wearenotwaiting"-Bewegung, initial in den USA entstanden, sind inzwischen Systeme hervorgekommen, die mit vielen Insulinpumpen und Glucosesensoren kompatibel sind. Allerdings handelt es sich auch um ein Verlassen des CE-Labels der einzelnen Produkte, sodass die Herstellerverantwortung erlischt. Wenn Ärzte dieses aktiv unterstützen, kann es im Fall von Haftungsfragen sogar so sein, dass der Verordner als allein verantwortlich für das Produkt gilt. Aktuell ist kein Fall bekannt, in dem nachträglich ein Arzt verklagt wurde; dieses theoretische Risiko hat aber die Deutsche Diabetes Gesellschaft zu einer schriftlichen Stellungnahme veranlasst, die im praktischen Alltag allerdings nicht weiterhilft. Letztlich liegt das Dilemma bei den Ärzten: Wenn Patienten ein Open-Source-System nutzen, muss ihnen trotzdem Insulin verschrieben werden. Dieses zu verweigern, würde nicht nur ein u.U. langjähriges Vertrauensverhältnis kompromittieren, sondern auch dem „Sankt-Florian-Prinzip" entsprechen: Das Dilemma würde weitergeschoben, denn ein Mensch mit Typ-1-Diabetes braucht nun mal Insulin. Davon „nichts zu wissen“, kann letztlich kein Argument sein, denn der Verordner von Glucosesensoren ist auch dafür verantwortlich, die ge- nerierten Daten mit den Anwendern zu evaluieren.

॥) Aus juristischen Gründen sollten Ärzte keine Empfehlung für OpenSource-Systeme aussprechen

Beruhigend ist an dieser Stelle, dass sich die Open-Source-Systeme als hocheffektiv erwiesen haben. Es existieren bisher nur kleinere, abgeschlossene Studien [21], die solche Systeme als ebenso effektiv wie die kommerziellen Systeme bestätigen (- Abb. 1). Allerdings ist das Einrichten eines Open-Source-Systems auch kein Selbstläufer und primär für Menschen mit ausgeprägtem technischen Verständnis geeignet. Empfehlen sollten behandelnde Ärzte diese Systeme aus juristischer Sicht nicht.

\section{Therapieziele werden auf Technologie ausgerichtet}

Der $\mathrm{HbA}_{1 \mathrm{c}}$-Wert als einziger in Langzeitstudien validierter Wert ist seit der DCCT(Diabetes Control and Complications Trial)-Studie der Parameter, mit dem die glykämische Einstellung der letzten 8 bis 10 Wochen beurteilt wird. Ein höherer $\mathrm{HbA}_{1 \mathrm{c}}$-Wert geht mit einem höheren Risiko für den Eintritt von Folgeerkrankungen einher; ab $8 \%$ ( $64 \mathrm{mmol} / \mathrm{mol})$ steigt das Risiko exponentiell. Ein niedrigerer $\mathrm{HbA}_{1 c^{-}}$ Wert ist nicht mehr gleichbedeutend mit dem Risiko für mehr schwere Unterzuckerungen, wie dies in der Vergangenheit der Fall war [2]. Daher ist der $\mathrm{HbA}_{1 c}$-Wert weiterhin fester Bestandteil in der Regelversorgung und der Qualitätssicherung im Rahmen des Disease-ManagementProgramms.

Da aber der $\mathrm{HbA}_{1 c}$-Wert keine Aussage über die Durchführung, Probleme mit der Therapie, der Insulindosierung oder schwankenden Werten gibt, ist dieser Parameter zur Therapieanpassung und Beratung ungeeignet. Mithilfe des CGM entfällt bis auf wenige spezielle Situationen die Notwendigkeit, ein ausführliches Blutzuckertagebuch zu führen. Dieses ist nicht nötig, da Insulin- und Kohlenhydratmengen bei Nutzung eines Bolusrechners automatisch aufgezeichnet werden; die glykämischen Daten werden direkt über den Sensor erfasst. Somit kann die Beratung 
Hier steht eine Anzeige.

黑 Springer 
auf Basis dieser Daten, die in visualisierter oder numerischer Form das „echte Leben“ abbilden, erfolgen. Aus kumulierten Daten lasst sich ein Glucose Management Index (GMI) ableiten, der dem gemessenen $\mathrm{HbA}_{1 c}$-Wert recht nahekommt [22].

Die Zeit im Zielbereich kann ebenfalls errechnet werden; sie entspricht der Dauer, in der die Glucosekonzentration zwischen 70 und $180 \mathrm{mg} / \mathrm{dl}(3,9-10,0 \mathrm{mmol} / \mathrm{l})$ beträgt. Dieser Korridor ist in einem internationalen Konsens als der sinnvolle, permissive Stoffwechselbereich angesehen worden. Die Zeit im Zielbereich sollte über $70 \%$ des Tages erreicht werden, dann lässt sich ein $\mathrm{HbA}_{1 c}$-Wert < $7 \%$ (53 $\left.\mathrm{mmol} / \mathrm{mol}\right)$, wie in den Leitlinien vorgesehen, erreichen. Um unangenehme Situationen zu vermeiden, sollte die Dauer im hypoglykämischen Bereich $<70 \mathrm{mg} / \mathrm{dl}(3,9 \mathrm{mmol} / \mathrm{l})$ weniger als $4 \%$ des Tages betragen, also weniger als eine Stunde täglich [23]. Zur Vermeidung schwerer Unterzuckerungsereignisse sollten Werte $<54 \mathrm{mg} / \mathrm{dl}(3,0 \mathrm{mmol} / \mathrm{l})$ in weniger als $1 \%$ der Zeit auftreten. Die Zeit im Zielbereich korreliert hinsichtlich des Folgeerkrankungsrisikos mit dem $\mathrm{HbA}_{1 c^{-}}$ Wert [24].

\section{Fazit für die Praxis}

- In der pädiatrischen Behandlung des Typ1-Diabetes ist die Nutzung von Technologien wie Glucosesensor und Insulinpumpe Standard. Das Zusammenspiel von beiden kann eine Automatisierung der Insulinabgabe ermöglichen, die sich in der Insulindosierung stetig dem aktuellen Bedarf anpasst.

- Gesetzlich krankenversicherten Kindern und Jugendlichen steht derzeit nur ein „Automated-insulin-delivery“(AID)-System zur Verfügung.

- Für Kleinkinder gibt es keine regelhafte Versorgung mit interaktiven Systemen.

- Vollautomatische Systeme haben sich derzeit in der postprandialen Phase als nichteffektiv erwiesen und sind daher nicht marktreif.

- Eine strukturierte Schulung bleibt Grundlage einer anhaltenden und erfolgreichen Therapiedurchführung.
Korrespondenzadresse

\section{Dr. T. Biester}

AUF DER BULT, Kinder- und Jugendkrankenhaus Janusz-Korczak-Allee 12, 30173 Hannover, Deutschland

Biester@hka.de

\section{Einhaltung ethischer Richtlinien}

Interessenkonflikt. T. Biester hat Honorare für Vorträge oder Beratertätigkeit AstraZeneca, DexCom, Medtronic, Novo Nordisk, Roche, Sanofi, Ypsomed erhalten. Seit 2021 ist er Mitglied im Expertengremium für Medizinprodukte der EU-Kommission. Er ist Prüfarzt einer Studie mit Medtronic Insulinpumpen. K. Dovc hat Honorare für Vorträge von Pfizer, Novo Nordisk und Eli Lilly erhalten. A. Chobot hat Honorare für Vorträge von Medtronic, Novo Nordisk und Sanofi erhalten. M. Tauschmann hat Honorare für Vorträge von Novo Nordisk erhalten. Er ist Prüfarzt einer CamAPsStudie in Österreich. T. Biester und T. Kapellen sind Mitglieder im EXPAMED-Panel der EU-Kommission für Medizinprodukte.

Für diesen Beitrag wurden von den Autoren keine Studien an Menschen oder Tieren durchgeführt. Für die aufgeführten Studien gelten die jeweils dort angegebenen ethischen Richtlinien.

\section{Literatur}

1. Cherubini V, Grimsmann JM, Akesson K, Birkebaek NH, Cinek O, Dovc K et al (2020) Temporal trends in diabetic ketoacidosis at diagnosis of paediatric type 1 diabetes between 2006 and 2016: results from 13 countries in three continents. Diabetologia 63(8):1530-1541

2. Karges B, Rosenbauer J, Kapellen T, Wagner VM, Schober E, Karges W et al (2014) Hemoglobin A1c Levels and risk of severe hypoglycemia in children and young adults with type 1 diabetes from Germany and Austria: a trend analysis in a cohort of 37,539 patients between 1995 and 2012. PLoSMed 11(10):e1001742

3. Kamrath C, Mönkemöller K, Biester T, Rohrer TR, Warncke K, Hammersen J et al (2020) Ketoacidosis in children and adolescents with newly diagnosed type 1 diabetes during the COVID-19 pandemic in Germany. JAMA 324(8):801-804

4. Holder M, Ehehalt S (2020) Significant reduction of ketoacidosis at diabetes onset in children and adolescents with type 1 diabetes-The Stuttgart Diabetes Awareness Campaign, Germany. Pediatr Diabetes 21(7):1227-1231

5. Vliebergh J, Lefever E, Mathieu C (2021) Advances in newer basal and bolus insulins: impact on type 1 diabetes. Curr Opin Endocrinol Diabetes Obes 28(1):1-7

6. van den Boom L, Karges B, Auzanneau M, RamiMerhar B, Lilienthal E, von Sengbusch $S$ et al (2019) Temporal trends and contemporary use of insulin pump therapy and glucose monitoring among children, adolescents, and adults with type 1 diabetes between 1995 and 2017. Diabetes Care 42(11):2050-2056

7. Tauschmann M, Hermann JM, Freiberg C, Papsch M, Thon A, Heidtmann B et al (2020) Reduction in diabetic ketoacidosis and severe hypoglycemia in pediatric type 1 diabetes during the first year of continuous glucose monitoring: a multicenter analysis of 3,553 subjects from the DPV registry. Diabetes Care 43(3):e40-e42

8. Kamrath C, Tittel SR, Kapellen TM, von dem Berge T, Heidtmann B, Nagl K et al (2021) Early versus delayed insulin pump therapy in children with newly diagnosed type 1 diabetes: results from the multicentre, prospective diabetes follow-up DPV registry. Lancet Child Adolesc Health 5(1):17-25

9. Weisman A, Bai J-W, Cardinez M, Kramer CK, Perkins BA (2017) Effect of artificial pancreas systems on glycaemic control in patients with type 1 diabetes: a systematic review and meta-analysis of outpatient randomised controlled trials. Lancet Diabetes Endocrinol 5(7):501-512

10. Doyle FJ 3rd, Huyett LM, Lee JB, Zisser HC, Dassau E (2014) Closed-loop artificial pancreas systems: engineering the algorithms. Diabetes Care 37(5):1191-1197

11. Bergenstal RM, Garg S, Weinzimer SA, Buckingham BA, Bode BW, Tamborlane WV et al (2016) Safety of a hybrid closed-loop insulin delivery system in patients with type 1 diabetes. JAMA 316(13):1407-1408

12. Tauschmann M, Thabit H, Bally L, Allen JM, Hartnell S, Wilinska ME et al (2018) Closed-loop insulin delivery in suboptimally controlled type 1 diabetes: a multicentre, 12-week randomised trial. Lancet 392(10155):1321-1329

13. Breton MD, Kanapka LG, Beck RW, Ekhlaspour L, Forlenza GP, Cengiz E et al (2020) A randomized trial of closed-loop control in children with type 1 diabetes. NEngl J Med 383(9):836-845

14. Amadou C, Franc S, Benhamou PY, Lablanche S, Huneker E, Charpentier G et al (2021) Diabeloop DBLG1 closed-loop system enables patients with type 1 diabetes to significantly improve their glycemic control in real-life situations without serious adverse events: 6-month follow-up. Diabetes Care 44(3):844-846

15. NimriR, Bratina N, Kordonouri O, AvbeljStefanija M, Fath M, Biester T et al (2017) MD-Logic overnight type 1 diabetes control in home settings: a multicentre, multinational, single blind randomized trial. Diabetes Obes Metab 19(4):553-561

16. Dovc K, Battelino T (2021) Time in range centered diabetes care. Clin Pediatr Endocrinol 30(1):1-10

17. Messer LH, Berget C, Vigers T, Pyle L, Geno C, Wadwa RP et al (2020) Real world hybrid closedloop discontinuation: predictors and perceptions of youth discontinuing the $670 \mathrm{G}$ system in the first 6 months. Pediatr Diabetes 21(2):319-327

18. Biester T, Bratina N, Lange K, Biester S, Remus K, Thomas A et al (2020) Diabetesberatung zum Hybrid-AID-System bei Typ-1-Diabetes: neue Perspektiven und Therapieempfehlungen. Diabetol Stoffwechs 15(02):147-156

19. Bergenstal RM, Nimri R, Beck RW, Criego A, Laffel L, Schatz D et al (2021) A comparison of two hybrid closed-loop systems in adolescents and young adults with type 1 diabetes (FLAIR): a multicentre, randomised, crossover trial. Lancet 397(10270):208-219

20. Forlenza GP, Cameron FM, Ly TT, Lam D, Howsmon DP, Baysal Netal (2018) Fully closed-loop multiple model probabilistic predictive controller artificial pancreas performance in adolescents and adults in a supervised hotel setting. Diabetes Technol Ther 20(5):335-343

21. Kesavadev J, Srinivasan S, Saboo B, Krishna BM, Krishnan G (2020) The do-it-yourself artificial pancreas: a comprehensive review. Diabetes Ther 11(6):1217-1235 
22. Bergenstal RM, Beck RW, Close KL, Grunberger G, Sacks DB, Kowalski A et al (2018) Glucose management indicator (GMI): a new term for estimating $\mathrm{A} 1 \mathrm{C}$ from continuous glucose monitoring. Diabetes Care 41(11):2275-2280

23. Battelino T, Danne T, Bergenstal RM, Amiel SA, Beck R, Biester T et al (2019) Clinical targets for continuous glucose monitoring data interpretation: recommendations from the international consensus on time in range. Diabetes Care 42(8):1593-1603

24. Beck RW, Bergenstal RM, Riddlesworth TD, Kollman C, Li Z, Brown AS et al (2019) Validation of time in range as an outcome measure for diabetes clinical trials. Diabetes Care 42(3):400-405

25. Brown SA, Kovatchev BP, Raghinaru D, Lum JW, Buckingham BA, Kudva YC et al (2019) Sixmonth randomized, multicenter trial of closedloop control in type 1 diabetes. N Engl J Med 381(18):1707-1717

26. Benhamou P-Y, Franc S, Reznik Y, Thivolet $C$, Schaepelynck P, Renard E et al (2019) Closed-loop insulin delivery in adults with type 1 diabetes in real-life conditions: a 12-week multicentre, openlabel randomised controlled crossover trial. Lancet Digit Health 1(1):e17-e25

27. Petruzelkova L, Jiranova P, Soupal J, Kozak M, Plachy L, Neuman V et al (2021) Pre-school and school-aged children benefit from the switch from a sensor-augmented pump to an AndroidAPS hybrid closed loop:a retrospective analysis. Pediatr Diabetes 22(4):594-604

28. Gawrecki A, Zozulinska-Ziolkiewicz D, Michalak MA, Adamska A, Michalak M, Frackowiak U et al (2021) Safety and glycemic outcomes of do-it-yourself AndroidAPS hybrid closed-loop system in adults with type 1 diabetes. PLoS ONE 16(4):e248965

29. Biester T, Dovc K, Chobot A, Tauschmann M, Kapellen T (2021) AID (Automated Insulin Delivery) Systeme in der Diabetologie. Diabetologe. https:// doi.org/10.1007/s11428-021-00777-1

\section{Individualization of diabetes treatment by automated insulin delivery}

Insulin pumps and glucose sensors have been shown to be effective in improving diabetes treatment and reducing acute complications according to data from registries. Therefore, in pediatric diabetology the use of at least one technical device is standard. Both devices can also be combined to form automated insulin delivery (AID) systems. Many AID systems have been tested in clinical trials and have proven to be safe and effective. The supply situation in Germany currently only allows one system to be prescribed for people insured by the statutory health insurances. Currently, children younger than 7 years of age cannot be treated with this system. The reasons for this are legal hurdles and lack of certification by the manufacturers. The CE certification can also lead to problems with insulin prescriptions. Open-source systems are nonregulated variants to circumvent existing regulatory conditions. There are risks here for both users and prescribers.

For permanent use a thorough knowledge of the features of each AID system is necessary for both the user and the practitioner. Complete automation does not yet work. For the evaluation of the AID treatment, the metric data of the glucose sensors, the time in range and the glucose management index are the recognized and suitable parameters, because they allow a consultation based on real data from the daily life of people with diabetes.

As all glucose sensors are read out via cloud-based software or the data are obtained directly and automatically from a telephone-linked receiver device, this provides the ideal technical basis for telemedical care, which still needs to be configured.

\section{Keywords}

Insulin pump - Glucose sensor - Insulin infusion systems - Time in range - Telemedicine 